\title{
COMPLETELY ADDITIVE MEASURE AND INTEGRATION
}

\author{
ALAN McK. SHORB
}

ABSTRACT. This paper is an extension of the efforts to cast the theory of measure and integration into the framework of nonstandard analysis, begun by Robinson [7, particularly Theorem 3.5.2], and continued by Bernstein and Wattenberg, Loeb and Henson. The principal result, Theorem 3, is: There exists a completely additive measure function defined on all subsets of $R$ which nearly agrees with Lebesgue measure and is nearly translation invariant on bounded sets. Its integral is defined for all sets and functions, and nearly agrees with the Lebesgue integral.

1. Introduction. The infinitely small and infinitely large numbers of Robinson's nonstandard analysis [7] suggest that one could develop a theory of measure and integration which avoids some of the troublesome features of Lebesgue integration, such as nonempty sets with zero measures and nonmeasurable sets. This paper is an extension of the work already done in this direction ([3], [6], [5], [2] and [1]).

The ultimate theory should revolve around a nonstandard-valued measure function which enjoys the following properties:

(1) Every nonempty set has a positive, nonstandard measure, which nearly agrees with Lebesgue measure whenever the latter exists.

(2) The integral of any function over any set is defined and is computed by multiplying the measure of each point times the value of the function and summing over all points in the set. This integral nearly agrees with the Lebesgue integral whenever the latter exists.

(3) The measure is translation invariant.

(4) The measure is completely additive in the sense that the measure of the union of any disjoint family of sets is the sum of the measures of the sets in that family.

The three theorems of this paper are concerned with developing such a measure which enjoys the last three of these properties.

We assume a knowledge of measure and integration as found in [8], and a knowledge of nonstandard analysis as found in Chapters 2 and 3 of [7]. We use a notation scheme for sums and unions illustrated by:

Presented to the Society, January 28, 1973 under the title $A$ completely additive nonstandard measure function on $R$; received by the editors September 24, 1974. AMS (MOS) subject classifications (1970). Primary 02H25, 26A98, 26A60; Secondary $28 \mathrm{~A} 10,28 \mathrm{~A} 25,28 \mathrm{~A} 70$. 


$$
\sum f_{a}:(a \in A) \equiv \sum_{a \in A} f_{a}
$$

$(X, B, \mu)$ will denote a $\sigma$-finite measure space where $X=U X^{i}:(1 \leq i<\infty)$, $\mu\left(X^{i}\right)<\infty$ and $X^{i} \subseteq X^{i+1}$. If $A \subseteq X$, we denote $A \cap X^{i}$ by $A^{i}$. We also assume that $B$ separates points of $X$ in the sense that if $a \neq b \in X$, then there is a set $A \in \mathbb{B}$ with $a \in A, b \notin A$. We will let $(R, \mathbb{B}, \lambda)$ denote the reals with Lebesgue measure. All functions considered are finite valued.

If $A$ is a * finite set, we denote by $\# A$ the number of elements in $A$. A sample on the standard set $A$ is a ${ }^{*}$ finite set $A \subseteq S \subseteq{ }^{*} A$.

Fact. For any standard set $A$ there is a sample. A presample on $A$ is a finite subset of $A$ used to construct a sample on $A$. If $n \epsilon^{*} N, N_{n}=$ $\left\{m \in \epsilon^{*} N \mid 0<m \leq n\right\}$.

Let $\lambda^{\prime}$ be a ${ }^{*} R$ valued set function on $\mathcal{P}(R)$. We shall say that $\lambda^{\prime}$ nearly agrees with $\lambda$ if for every $A \in \mathcal{B}$ with $\lambda(A)$ defined, $\lambda^{\prime}(A) \simeq \lambda(A)$. $\lambda^{\prime}$ is nearly translation invariant on $A$ if for every $r \in R, \lambda^{\prime}(A) \simeq \lambda^{\prime}(A+r)$.

2. Integration. Let $A \subseteq X$. A partition of $A$ is a disjoint family $\mathcal{P}$ of subsets of $A$ such that $\cup \mathcal{P}^{-}=A$. Note that every simple function determines a partition. A pointed partition of $A$ is an ordered pair $(\mathcal{P}, S)$ such that $\mathcal{P}$ is a partition of $A$ and $S$ is a subset of $A$ which meets each member of $\mathcal{P}$ in exactly one point. All partitions in this paper will be assumed indexed. If $(\mathcal{P}, S)$ is a pointed partition, and $P_{j} \in \mathcal{P}$, we let $s_{j}$ denote the unique member of $P_{j} \cap S$. For any set $B \subseteq A$, we let $I(B)=\left\{1 \leq j \leq I \mid s_{j} \in B\right\}$. A pointed partition $\left(\mathcal{P}^{\prime}, S^{\prime}\right)$ is a refinement of the pointed partition $(\mathcal{P}, S)$ if $\mathcal{P}^{\prime}$ is a refinement of $\mathcal{P}$ and if $S^{\prime} \supseteq S . \mathcal{P}$ is called measurable if $\mathcal{P} \subseteq \mathcal{B}$.

Theorem 1. Let $(X, B, \mu)$ be a $\sigma$-finite measure space. Then there exists an integer $M \in \epsilon^{*} N-N$ and $a^{*}$ finite measurable pointed partition $(\mathcal{P}, S)$ of $X^{M}$ such that:

(*a) $S$ is a sample on $X^{M}$.

( $\left.{ }^{*} \mathrm{~b}\right)$ For every $A \in \mathbb{B}_{,}{ }^{*} A^{M}=\bigcup P_{j}:\left(j \in I\left({ }^{*} A\right)\right)$.

( ${ }^{*}$ c) If $f$ is integrable over $A$,

$$
\int_{A} f d \mu={ }^{\circ} \sum f\left(s_{j}\right) \cdot{ }^{*} \mu\left(P_{j}\right):\left(j \in I\left({ }^{*} A\right)\right) .
$$

Proof. This follows from

Lemma 1 (Fnabling lemma). Let $\epsilon>0$. Let $a_{1}, \ldots, a_{n} \in X, A_{1}, \ldots, A_{p}$ $\epsilon:$ and $f_{1}, \ldots, f_{q}$ be measurable functions. Then there exist a natural number $M$ and a finite measurable pointed partition $(\mathscr{P}, S)$ of $X^{M}$ such that:

(a) $a_{i} \in S, 1 \leq i \leq n$.

(b) $A_{i}^{M}=\bigcup P_{j}:(j \in I(A))$.

(c) If $f_{l}$ is integrable over $\Lambda_{i}$, then 


$$
\left|\int_{A_{i}} f_{l} d \mu-\sum f_{l}\left(s_{j}\right) \cdot \mu\left(P_{j}\right):\left(j \in I\left(A_{i}\right)\right)\right|<\epsilon .
$$

Proof. We may assume that each $f_{l} \geq 0$ by considering positive and negative parts. We may assume that $f_{l}$ is integrable over all of $X$ by multiplying it by $\chi\left(A_{i}\right)$. We may assume the $a_{i}$ 's are separated by adding new $A_{i}^{\prime}$ 's.

Let $f$ be the sum of the $f_{l}$ 's. Choose $M$ so that each $a_{i} \in X^{M}$ and

$$
\left|\int_{X} f d \mu-\int_{X M} f d \mu\right|<\epsilon / 5 \text {. }
$$

Choose $B \subseteq X^{M}$ so that $f$ is bounded on $B, a_{i} \in B$, and

$$
\left|\int_{X M} f d \mu-\int_{B} f d \mu\right|<\epsilon / 5 \text {. }
$$

On $B$, approximate each $f_{l}$ from below by a simple function $f_{l}^{\#}$ such that

$$
\begin{gathered}
\mu(B)\left(f_{l}(x)-f_{l}^{\#}(x)\right)<\epsilon / 5 \text { for all } x \in B, \\
\int_{B}\left(f_{l}-f_{l}^{\#}\right) d \mu<\epsilon / 5 .
\end{gathered}
$$

The partition of $X^{i 1}$ will be the common refinement of the partitions deter-

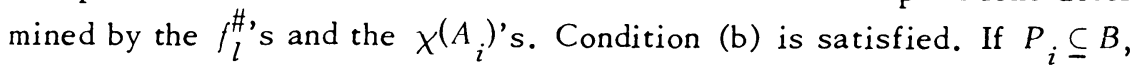
choose $s_{i}$ in any manner, as long as (a) is satisfied. If $P_{i} \subseteq X^{M}-B$, choose $s_{i}$ so that

$$
f\left(s_{i}\right)-\epsilon / 5<\min \left\{f(x) \mid x \in P_{i}\right\} .
$$

Then equations (1)-(5) assure that condition (c) is satisfied, and the lemma is proved.

Proof of theorem. Let $Q(\alpha, M)$ be the relation where $\alpha=\langle A, f, a, \epsilon\rangle$, as in the lemma, and where $X^{M}$ has a partition as in the lemma. Then $Q$ is concurrent, and the theorem is proved.

Corollary. For any positive infinitesima: $\epsilon$, there is an integer $M \epsilon^{*} N$ with $a{ }^{*}$ finite measurable pointed partition satisfying $\left({ }^{*} \mathrm{a}\right),\left({ }^{*} \mathrm{~b}\right)$ and $(\mathrm{c})$.

Proof. The following relation is concurrent. $Q^{\prime}(\alpha, \beta)$, where $\alpha=$ $\langle A, f, a\rangle$ as before, and $\beta: R^{+} \rightarrow N$ such that if $\beta(\epsilon)=1$, then $Q(\langle A, f, a, \epsilon\rangle, M)$. The corollary is proved.

3. Translation invariance. The pointed partition $(\mathcal{P}, S)$ of $X^{M}$ obtained in Theorem 1 allows us to define a measure function $\mu^{\prime}$ on $X$ which nearly agrees with $\mu$ in both measure and integration. We first define $\mu^{\prime}$ on all of $* \mathcal{P}(X)$, and then for $A \in P(X)$ identify $\mu^{\prime}(A)$ with $\mu^{\prime}(* A)$. For 
$A \in * \mathscr{P}(X)$ we let $\mu^{0}(A)=\Sigma^{*} \mu\left(P_{j}\right):(j \in I(\Lambda)) . \mu^{0}$ is seen to be a ${ }^{*}$ finitely additive set function, and if we let $f$ be the function which is identically 1 on $A \in B$, condition $\left({ }^{*} \mathrm{c}\right)$ of Theorem 1 states that $\mu^{\prime}$ agrees with $\mu$. If we define

$$
\left.\left.\int_{A} f d \mu^{\prime}=\sum f\left(s_{j}\right) \mu^{(p)}\right):\left(j \in I^{*} A\right)\right)
$$

for every $A \subseteq X$ and every $f: A \rightarrow R$, condition $\left({ }^{*} \mathrm{c}\right)$ states that $\mu^{\prime}$ nearly agrees with $\mu$ in integration.

In the special case of $(R, \beta, \lambda)$, we are not yet assured that the measure $\lambda^{\prime}$ as constructed in Theorem 1 is nearly translation invariant on bounded sets. The next theorem shows it can be.

Theorem 2. Let $(R, B, \lambda)$ be Lebesgue measure. Then $M_{r} \mathcal{P}$, and $S$ of Theorem 1 can be chosen so that $\lambda^{\prime}$ is nearly translation invariant on bounded sets.

Proof. Let $M, B$ and $\left(\mathcal{P}^{\circ}, S^{\prime}\right)$ be as assured in the lemma, with the additional property that all members of $\mathcal{P}^{\prime}$ with zero measure are subsets of $D=[-M, M]-B$, and that $\lambda(D)$ is small. The problem is to choose a refine-

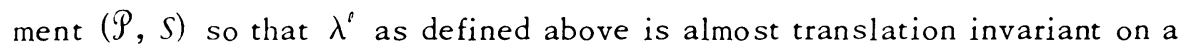
few given subsets of $[-M, M]$, without losing the properties assured by the lemma. The solution is to mimic the construction of [3], obtaining a presample $S \supseteq S^{\prime}$ on $[-M, M]$ such that if we define $\lambda_{S}(A)=2 M \cdot \#(A \cap S) / \# S$, then $\lambda_{S}(D)$ is very small, $\lambda(P) / \lambda_{S}(P)$ is almost 1 for every $B \supseteq P \in \mathcal{P}^{\prime}$, and $\lambda_{S}$ is almost translation invariant on the given subsets. We now need to encase $S$ in a refinement of $\rho^{\prime}$. Encase the new members of $S$ which belong to $D$ in singletons. For each $B \supseteq P \in \mathcal{P}^{\circ}$, if $\#(P \cap S)=q$, break $P$ into $q$ sets, each with the same $\lambda$ measure, and each with one point of $S$. Now we have a partition where all except a few of the sets have nearly the same $\lambda$ and $\lambda_{S}$ measure, so $\lambda^{\prime}$ as defined above is almost translation invariant. Since we have chosen the new partition judiciously, we have not lost any of the properties (a)-(c) inherited from the lemma. The theorem is proved.

4. Complete additivity. Let $A$ be a standard set. A counting function on $A$ is an internal function $C: *\left(P(A) \rightarrow{ }^{*} N\right.$ such that: (a) For every $a \in A$, $C\left(\left\{{ }^{*} a\right\}\right)=1$; (b) if $B$ and $D$ are disjoint subsets of $A$, then $C\left({ }^{*}(B \cup D)\right)=$ $C\left({ }^{*} B\right)+C\left({ }^{*} D\right)$; (c) for every $B \subseteq A$, if $C\left({ }^{*} B\right)=n$, there is an internal function $E[B]: N_{n} \stackrel{1-1}{\longrightarrow} * B$ such that $B \subseteq \operatorname{ran}(E[B])$. We identify $C(B)$ with $C\left(^{*} B\right)$, and note that $E[B]$ "enumerates" the elements of $B$, and $C(B)$ is the result of that "count". If $S$ is a sample on $A$, define the function $C[S]$ : $* \mathcal{P}(A) \rightarrow{ }^{*} N$ by $C[S](B)=\#(B \cap S)$. 
Lemma 2. $C[S]$ is a counting function.

Proof. Clear.

We now define the concept of a completely additive set function $\sigma$. Intuitively speaking, for any pairwise disjoint family $\mathcal{F}$ of subsets of $A$, we would like to have $\sigma(\bigcup \mathfrak{F})=\Sigma \sigma(B):(B \in \mathcal{F})$. More formally, for any standard set $A$, a completely additive set function on $A$ is a function $\sigma:{ }^{*} \mathcal{P}(A) \rightarrow{ }^{*} R$, along with a counting function $C$ on $\mathcal{P}(A)$ such that for every pairwise disjoint family, $\mathcal{F}$, of subsets of $A$,

$$
\sigma\left(\bigcup^{*} \mathfrak{F}\right)=\sum \sigma(E[\mathcal{F}](i)):(1 \leq i \leq C(\mathcal{F})) .
$$

Theorem 3. There is a completely additive measure $\lambda^{\prime}$ on $R$ which nearly agrees with $\lambda$ in measure and integration and which is nearly trans. lation invariant on bounded sets.

(Note that in the statement of this theorem in [9], "translation invariant" should read "nearly translation invariant".)

Proof. This follows from Lemma 3 below. We first give some definitions.

Let $S$ be a sample on a standard set $X$. Let $\phi: S \rightarrow^{*} R$. Define $\sigma[\phi]$ : $* \mathcal{P}(A) \rightarrow{ }^{*} R$ by

$$
\sigma[\phi](\beta)=\sum \phi(s):(s \in S \cap \beta) .
$$

As above for $B \in \mathcal{P}(A)$, we identify $\sigma[\phi](B)$ with $\sigma[\phi]\left({ }^{*} B\right)$. If $f: B \rightarrow{ }^{*} R$ we define

$$
\int_{B} f d \sigma[\phi]=\sum \phi(s) f(s):(s \in B \cap s) .
$$

If $f$ and $B$ are standard, we identify $\int_{B} f d \sigma[\phi]$ with $\int_{*_{B}}{ }^{*} f d \sigma[\phi]$. Let $\mathbf{D}$ be the class of pairwise disjoint families of subsets of $A$, and let $\mathrm{S}$ be a sample on D. Let $\delta=\left\{B \in \in^{* \mathcal{P}}(A) \mid B=\varnothing\right.$ or $B \in \mathcal{F} \in \mathrm{S}$ and $\left.B \cap S \neq \varnothing\right\}$. Then $\mathcal{S}$ is a sample on $\mathcal{P}(A)$. For suppose $B \subseteq A$. If $B=\varnothing,{ }^{*} B \in \mathcal{S}$ by designation. If $b \in B$, then $b \in{ }^{*} B \cap S$ and ${ }^{*} B \in\left\{{ }^{*} B\right\} \in S$. Therefore ${ }^{*} B \in \mathcal{S}$. Also, $S$ has only ${ }^{*}$ finitely many members, and each of them has only ${ }^{*}$ finitely many members which meet $S$, so $S$ is ${ }^{*}$ finite.

Lemma 3. Let $S, \phi$ and $\mathcal{S}$ be as described above. Then $\sigma[\phi]$ is a completely additive function on $A$ with associated counting function $C[\Phi]$.

Proof. Let $\mathcal{F} \in \mathbf{D}$. Let $B=\bigcup \mathfrak{F}$. Let $n=\#(\mathcal{S} \cap * \mathfrak{F})=C[\mathcal{S}](\mathfrak{F})$, and let $E[\mathcal{F}]$ be the associated enumerating function. Now, $S \cap{ }^{*} B=S \cap$ $(\bigcup E[F](i):(1 \leq i \leq n))$. For suppose $s \in S \cap{ }^{*} B$. Then $s \in S \cap D$ for some $D \in * \mathfrak{F}$. Therefore $D \in \mathcal{S}$, so $D=E[\mathcal{F}](i)$ for some $1 \leq i \leq n$. The converse 
is clear. Furthermore, since $\mathcal{F}$ is pairwise disjoint, so is $\operatorname{ran}(E[\mathcal{F}])$. Therefore

$$
\begin{aligned}
\sigma[\phi]\left({ }^{*} B\right)= & \sum \phi(s):(s \in(\bigcup(S \cap E[\mathcal{F}](i)):(1 \leq i \leq n))) \\
= & \sum\left(\sum \phi(s):(s \in S \cap E[\mathcal{F}](i))\right):(1 \leq i \leq n), \\
& \quad \text { (because } \operatorname{ran}(E[\mathcal{F}]) \text { is disjoint) } \\
= & \sum \sigma[\phi](E[\mathcal{F}](i)):(1 \leq i \leq n) .
\end{aligned}
$$

The lemma is proved.

Proof of theorem. Let $(\mathcal{P}, S)$ be as in Theorem 2. Define $\phi\left(s_{i}\right)=$ ${ }^{*} \mu\left(P_{i}\right)$. Then $\sigma[\phi]=\lambda^{\prime}$, and the theorem follows from Theorem 2 and Lemma 3.

Now $\lambda^{\prime}$ is nearly translation invariant on bounded sets. We would expect $\lambda^{\prime}$ to be nearly translation invariant on any set with a finite $\lambda^{\prime}$ measure. We have not been able to show this. More generally, the above theorems do not tell us about the relative size of the translate of a set $A$ if $\lambda^{\prime}(A)$ is either infinite or infinitesimal. We shall say that $\lambda^{0}$ is proportionally trans. lation invariant if for every set $A$ with $\lambda^{\prime}(A) \neq 0, \lambda^{0}(B) / \lambda^{\prime}(A) \simeq 1$ for any translate $B$ of $A$.

Conjecture. $\lambda^{\prime}$ of the theorems can be assured of being proportionally translation invariant.

The concept of near translation invariance can be generalized. Let $(X, B, \mu)$ be a measure space, and let II be a group of permutations of $X$ such that for every $A \in B$ and every $\sigma \in \Pi$ we have $\mu(A)=\mu(\sigma(A))$. Then we shall say that $\mu$ is II-invariant. If $\mu^{\prime}$ is a completely additive measure on $X$ which agrees with $\mu$, we say $\mu^{\prime}$ is nearly II-invariant if for every $\alpha \in \Pi$ and $A \subseteq X, \mu^{\prime}(A) \simeq \mu^{\prime}(\sigma(A))$.

False conjecture. If $\mu$ is $\Pi$-invariant then there is a completely additive $\mu^{\prime}$ which agrees with $\mu$ and is nearly $\Pi$-invariant.

Proof. Let $X=R^{3}$ with $\mu$ Lebesgue measure, and let II be the group of rigid motions of $R^{3} \cdot \mu$ is II-invariant. Let $A$ be the example of Hausdorff $[4$, p. 469] which is at the same time $1 / 2$ and $1 / 3$ of the unit sphere by means of rigid rotations. If $\mu^{\prime}$ existed as in the conjecture, we would have that $2 \Pi / 3 \simeq \mu^{\circ}(A) \simeq 4 \Pi / 9$.

\section{BIBLIOGRAPHY}

1. Allen R. Bernstein, A nonstandard integration theory for unbounded functions, Z. Math. Logik Grundlagen Math. 20 (1974), 97-108.

2. Allen R. Bernstein and Peter A. Loeb, A nonstandard integration theory for unbounded functions, Victoria Symposium on Nonstandard Analysis, Lecture Notes in Math., vol. 369, Springer-Verlag, New York, 1974.

3. A. R. Bernstein and F. Wattenberg, Nonstandard measure theory, Applications of Model Theory to Algebra, Analysis and Probability (Internat. Sympos., Pasadena, Calif., 1967), Holt, Rinehart and Winston, New York, 1969, pp. 171-185. MR $40 \# 287$. 
4. F. Hausdorff, Grundzüge der Mengenlehre, Veit, Leipzig, 1914; photographic reproduction, Chelsea, New York, 1949. MR 11, 88.

5. C. Ward Henson, $O n$ the representation of measures, Trans. Amer. Math. Soc. 172 (1972), 437-446. MR $47 \# 3631$.

6. Peter A. Loeb, A nonstandard representation of measurable spaces, $L^{\infty}$ and $L_{\infty}^{*}$, Contributions to Nonstandard Analysis, W. A. J. Luxemburg and A. Robinson (Editors), North-Holland, Amsterdam, 1972, pp. 65-80.

7. Abraham Robinson, Non-standard analysis, North-Holland, Amsterdam, 1966. MR 34 \#5680.

8. H. L. Royden, Real analysis, Macmillan, New York, 1963. MR 27 \#1540.

9. A. M. Shorb, A completely additive nonstandard measure function on $R$, Notices Amer. Math. Soc. 20 (1973), A-32. Abstract \#701-02-9.

DEPARTMENT OF MATHEMATICS, NAVAL POSTGRADUATE SCHOOL, MONTEREY, CALIFORNIA 93940

Current address: System Dynamics Group, Sloan School, Massachusetts In stitute of Technology, Cambridge, Massachu setts 02139 